\title{
RSC y empresas tecnológicas: Un estudio sobre las motivaciones de implantación e integración en empresas
} españolas

CSR and technology companies: A study on the motivations of implementation and integration in Spanish companies

\author{
Juan Andrés Bernal Conesa
}

Dpto. de Ciencias Económicas y Jurídicas del Centro Universitario de la Defensa de San Javier (CUD). C/Coronel López Peña s/n. CP 30720, Santiago de la Ribera (Murcia). jandres.bernal@cud.upct.es

\section{Antonio Juan Briones Peñalver}

Dpto. Organización de Empresas de la Facultad de Ciencias de la Empresa de la Universidad Politécnica de Cartagena (UPCT), Calle Real, 3, CP 30201, Cartagena, Murcia. aj.briones@upct.es

\section{Carmen de Nieves Nieto}

Dpto. de Ciencias Económicas y Jurídicas del Centro Universitario de la Defensa de San Javier (CUD). C/Coronel López Peña s/n. CP 30720, Santiago de la Ribera (Murcia).carmen.denieves@upct.es

\section{Resumen}

En este artículo se propone un modelo de ecuaciones estructurales para explicar las motivaciones de implantar medidas de Responsabilidad Social Corporativa (RSC) en empresas tecnológicas españolas y cómo influyen en la integración de la RSC la presencia de sistemas de gestión normalizados previos a la implantación de dichas medidas. El análisis de la RSC en empresas tecnológicas se encuentra en un estado inicial como demuestra la literatura analizada. Se llevó a cabo un estudio en empresas ubicadas en parques científicos y tecnológicos españoles. Los resultados del modelo revelan que existe una relación positiva, directa y estadísticamente significativas entre las motivaciones, los sistemas de gestión previos, la implantación de medidas de RSC y la integración real de la RSC en la organización, y por tanto con implicaciones teóricas y prácticas para la gestión de la RSC en empresas tecnológicas cubriendo el hueco encontrado en la literatura.

Palabras clave: Responsabilidad social corporativa, motivaciones, empresas tecnológicas, integración, ecuaciones estructurales.

\section{Abstract}

In this article a structural equation model is proposed to explain the motivations of implementing Corporate Social Responsibility (CSR) measures in Spanish technology companies and its linkage with other standardized management systems before CSR implementation. The analysis of CSR in technology companies is in an initial state, as shown by the literature analyzed. The study was conducted in companies located in Spanish Science and Technology Parks. For this study, a survey was sent and structural equation model was used.

Model results show that there is a positive, direct and statistically significant relationship between the motivations, previous management systems, implementation of CSR and the real integration of CSR in the organization. The links between the motivations of CSR, CSR actions and their integration in technology companies are reliably and empirically demonstrated.

Keywords: Corporate social responsibility, motivation, technology companies, integration, structure equation modeling.

\section{Introducción}

Si algo caracteriza el entorno empresarial en los últimos años ha sido la aguda crisis económica sufrida, la cual no puede ser atribuida meramente a un cambio de ciclo económico sino también a la ausencia de valores y principios éticos en el funcionamiento de las organizaciones (Melé, Argandoña, \& Sanchez-Runde, 2011). A lo largo de este periodo se ha puesto de manifiesto la importancia de la transparencia y la ética como atributos estrechamente vinculados a la gestión empresarial. Por tanto, se puede decir que la Responsabilidad Social Corporativa (RSC) guarda una estrecha relación con los períodos de crisis económica, en el sentido de que la ausencia de la misma, se considera uno de los factores desencadenantes de los problemas financieros (Pérez Ruiz \& Rodríguez del Bosque, 2012), al tiempo que su gestión efectiva puede ayudar a las organizaciones a minimizar los impactos negativos de la crisis (Janssen, Sen, \& Bhattacharya, 2015).

Por otro lado, las organizaciones están constantemente adaptándose a los cambios económicos con la intención de tener mayores posibilidades de supervivencia en el mercado. Un factor clave para ello es la innovación (Bernardo, 2014). Ésta se puede contextualizar como "la adopción de una idea o un nuevo comportamiento en la organización" (Damanpour \&
Gopalakrishnan, 2001, pag.47). La RSC es una innovación en la gestión pues contribuye y fomenta: (1) la innovación que resulta del diálogo con los grupos de interés (stakeholders) tanto internos como externos a la empresa, (2) la identificación de nuevas oportunidades de negocio derivadas de las demandas sociales y medioambientales en productos y procesos más eficientes o en nuevas formas de negocio dirigidas a la denominada base de la pirámide, formada por las personas con menos recursos (Prahalad \& Ramaswamy 2004) y (3) la creación de mejores lugares y formas de trabajar que favorecen la innovación y la creatividad, como las basadas en mayor participación de los empleados y más confianza en ellos (Benito-Hernández \& Esteban-Sánchez, 2012).

Por ello, las empresas, deben adoptar prácticas formalizadas de RSC $y$, por tanto, establecer aquellos procedimientos $y$ herramientas que estén alineadas con su estrategia corporativa (Bocquet, Le Bas, Mothe, \& Poussing, 2013), pues la RSC se traduce en un mayor éxito competitivo (Herrera, Larrán, \& MartínezMartínez, 2013), siempre y cuando esté integrada en la organización y con ello genere una innovación en los proceso de gestión(Benito-Hernández \& Esteban-Sánchez, 2012). Si la RSC está integrada en los procesos de negocio genera prácticas innovadoras las cuales pueden ser fuente de una mejora de la competitividad (Vilanova, Lozano, \& Arenas, 2009). Dicha 
integración puede ser facilitada por sistemas de gestión normalizados previamente implantados.

Siendo conscientes de todas estas situaciones, el objetivo de este artículo es: (1) investigar las motivaciones de las empresas tecnológicas para tomar parte de las iniciativas de RSC e implantar actividades y políticas en su seno, integrándolas en el sistema de gestión propio de cualquier organización y (2) analizar los factores facilitadores de la implantación e integración de la RSC en el seno de la organización. Para ello el artículo se estructura con un apartado (2) de revisión de la literatura para conocer la situación de la RSC en empresas tecnológicas españolas, sus posibles motivaciones y cómo es la integración de la RSC en los sistemas de gestión de la empresa. A continuación se plantea el modelo conceptual y las hipótesis objeto de estudio (apartado 3). Un apartado (4) descriptivo de la metodología y técnica empleada para el estudio para mostrar los resultados obtenidos en el apartado 5. Para finalizar con un apartado (6) sobre la discusión, conclusiones y limitaciones del estudio.

\section{Revisión de la literatura}

Académicamente, la Responsabilidad Social Corporativa(RSC) se utiliza a menudo como un término general para describir una variedad de cuestiones relacionadas con las responsabilidades de las empresas (Hillenbrand, Money, \& Ghobadian, 2013). Sin embargo, no existe una definición universalmente aceptada sobre la RSC (Dahlsrud, 2008), aunque se puede afirmar que la RSC es, además del cumplimiento estricto de las obligaciones legales vigentes, la integración voluntaria en el gobierno y gestión, en la estrategia, políticas y procedimientos, de las preocupaciones sociales, laborales, medioambientales y de respeto a los derechos humanos que surgen de la relación y el diálogo transparente con sus grupos de interés, responsabilizándose así de las consecuencias y los impactos que se derivan de las acciones de una organización (Mendoza, De Nieves, \& Briones, 2010). Así pues, destaca en el estudio de la RSC una perspectiva teórica basada en la conocida Teoría de los Stakeholders, la cual establece que las empresas no sólo deben satisfacer a sus accionistas sino también a una amplia variedad de grupos que puedan afectar o verse afectados por las acciones de la empresa (Freeman \& Reed, 1983).

Por otro lado, en la literatura científica existen numerosos estudios sobre la RSC tanto en grandes empresas (Melé, Debeljuh, \& Arruda, 2006), como en pequeñas (Baumann-Pauly, Wickert, Spence, \& Scherer, 2013; Vázquez-Carrasco \& López-Pérez, 2013) $y$ en diferentes sectores (Bernal-Conesa, De Nieves-Nieto, \& Briones-Peñalver, 2014; Moseñe, Burritt, Sanagustín, Moneva, \& Tingey-Holyoak, 2013) e incluso alguno que hace referencia a empresas del sector tecnológico (Guadamillas-Gómez, DonateManzanares, \& Skerlavaj, 2010). Se encuentran además estudios sobre las posibles motivaciones de adoptar la RSC (Graafland \& Schouten, 2012) de forma general y su integración con otros sistemas de gestión de la empresa (Bernardo, 2014; Von Ahsen, 2014). Sin embargo, no se han encontrado estudios sobre la RSC, sus motivaciones e integración en empresas tecnológicas, las cuales son fuente constante de innovación, tanto en procesos como en productos, ya que el estudio del papel de las empresas tecnológicas en la gestión ambiental, la sostenibilidad y por tanto en la RSC está aún en sus etapas iníciales (Wang, Chen, \& BenitezAmado, 2015). De hecho para fomentar las innovaciones de producto y proceso, las empresas deben adoptar prácticas formalizadas de RSC (Boulouta \& Pitelis, 2014).

Diversos autores (Perrini, Russo, \& Tencati, 2007; Spence, 2007) han señalado el sector como uno de los elementos que inciden sobre la cultura organizativa a la hora de adoptar e integrar las prácticas de RSC en los planes estratégicos de las empresas. Así, por ejemplo, Perrini et al. (2007) detectaron como las empresas del sector de las Tecnologías de la Información y la Comunicación (TIC) estaban más predispuestas a controlar e informar sobre sus comportamientos de RSC mientras que las empresas manufactureras se interesaban más por motivar a sus empleados a través de actividades de voluntariado en la comunidad donde residen.

En determinados sectores tecnológicos, los períodos de desarrollo de productos son extremadamente largos y las empresas suelen presentar resultados negativos en los primeros años de vida, presentado mayores dificultades de financiación. En estos casos, los indicadores financieros no son efectivos para valorar el potencial de las empresas, siendo más adecuados los activos intangibles y los basados en el conocimiento (Quintana García, Benavides Velasco, \& Guzmán Parra, 2013).

Entre estos activos intangibles podemos encontrar la RSC, la cual como demuestran algunos estudios tiene una relación positiva con el performance (Muñoz, Pablo, \& Peña, 2015; Hammann, Habisch, $\&$ Pechlaner, 2009). Además, la actividad desarrollada por una empresa tiene un alto impacto social cuando ésta opera en el sector de las tecnologías de la información y las telecomunicaciones (Luna Sotorrío \& Fernández Sánchez, 2010), ya que se produce una generación de puesto de trabajo cualificados y fomenta la formación de trabajadores (JiménezZarco, Cerdán-Chiscano, \& Torrent-Sellens, 2013; VásquezUrriago, Barge-Gil, \& Rico, 2012).

Es por ello que se va a investigar y analizar la situación de las empresas tecnológicas españolas frente a la RSC, tomando como punto de partida las ubicadas en parques científicos y tecnológicos españoles pues en ellos hay una mayor presencia de empresas de alta tecnología (Vásquez-Urriago et al., 2012).

\subsection{Sector tecnológico español y RSC}

Las empresas ubicadas en los parques científicos y tecnológicos (PCyT) muestran un mayor esfuerzo en innovación y valoran más los obstáculos a la innovación (Vásquez-Urriago, Barge-Gil, Rico, \& Paraskevopoulou, 2014) y se aprecia un nivel de cooperación significativamente mayor en el caso de estas empresas (VásquezUrriago et al., 2012).

El concepto de parque científico se originó a finales de 1950 en el contexto universitario estadounidense (Jiménez-Zarco et al., 2013). El éxito de parques tecnológicos como el de Silicon Valley en California o el de Cambridge en Reino Unido ha influido para reproducir el modelo en otros países (Ratinho \& Henriques, 2010).

En España, los primeros parques surgieron a mediados de la década de 1980, siguiendo una estrategia de atracción de empresas de alta tecnología (Jiménez-Zarco et al., 2013) con el objetivo de contribuir al crecimiento económico y empresarial en el ámbito local o regional. Actualmente existen 68 parques científicos y tecnológicos y en ellos se acogen a empresas de 
origen, naturaleza e intereses distintos: spin-offs académicas de Base Tecnológica (EBT), Empresas de Base de Conocimiento (EBC) y start-ups (Jiménez-Zarco et al., 2013).

Los PCyT tienen en común la creación de empresas tecnológicas o atraer a empresas ya consolidadas para fomentar el desarrollo regional a través de un enfoque tecnológico y la creación de empleo y bienestar (Ratinho \& Henriques, 2010; Jiménez-Zarco et al., 2013). Por tanto, los parques tecnológicos estarían directamente relacionados con dos de las tres dimensiones de la RSC (la económica y la social) y generarían una red de cooperación entre empresas tecnológicas, lo cual puede aumentar la capacidad de general conocimiento y ampliar positivamente las relaciones con los agentes propios del negocio.

Además, las empresas pertenecientes a los parques son en promedio de menor tamaño, tienen una mayor vocación exportadora, pertenecen más frecuentemente a un grupo de empresas, son en mayor proporción de reciente creación y tienen una disminución menos frecuente de la cifra de negocios por venta o cierre de la empresa (Vásquez-Urriago et al., 2012).

Como se puede observar las empresas tecnológicas juegan un papel fundamental pues aportan inversión directa, generan empleos y ofrecen formación. Pero esta actividad también ha generado importantes retos sociales y laborales.

\subsection{Motivaciones de la RSC y su integración}

A pesar de la dificultad de aportar una definición de RSC (Dahlsrud, 2008), la idea general que se extrae de todas ellas es que la RSC supone que las empresas deben llevar a cabo sus negocios de manera que demuestren consideración para el más amplio entorno social con el fin de servir de manera constructiva a las necesidades de la sociedad.

Las motivaciones para la puesta en práctica de la RSC han sido estudiadas de forma general (Graafland \& Schouten, 2012), en diferentes países (Prajogo, Tang, \& Lai, 2012) y sectores, encontrándose dos tipos diferentes de motivaciones principales: las externas o extrínsecas a la organización, entre ellas destacan las de carácter financiero o económico que tienen relación con el beneficio (Graafland \& Schouten, 2012) y las internas o intrínsecas a la organización, que no solo tienen que ver con el beneficio de la organización sino también con los valores y creencias del personal que conforman las organizaciones (Graafland \& Schouten, 2012).

Para cumplir con los requisitos de los grupos de interés de un modo sistemático, las organizaciones emplean diferentes sistemas de gestión, entre los que cabe destacar la gestión de la calidad, el medio ambiente, la seguridad y salud laboral, y la responsabilidad social corporativa (Asif, Fisscher, Bruijn, \& Pagell, 2010). Dichos sistemas aparecen con frecuencia en la estrategia y políticas de empresa, y además las organizaciones están aplicando no sólo uno, sino una serie de estándares de sistemas de gestión para satisfacer sus propias necesidades así como las de los grupos de interés (Simon, Karapetrovic, \& Casadesus, 2012). Sin embargo en muchas empresas, la calidad, la salud y seguridad y la gestión ambiental existen como tres sistemas paralelos (Hamidi, Omidvari, \& Meftahi, 2012). Por lo tanto, un sistema de gestión integrado (SGI) debe integrar en la actualidad los aspectos (1) centrados específicamente en la calidad, la salud y seguridad, el medio ambiente, el personal y las finanzas y (2) de manera general en los grupos de interés y la responsabilidad para con dichos grupos, asumiendo por tanto diferentes niveles de integración (Jørgensen, Remmen, \& Mellado, 2006).

La necesidad de la integración de los sistemas de gestión individuales tiene sus raíces en la necesitad de utilizar eficazmente los recursos de la organización (Asif, Searcy, Zutshi, \& Fisscher, 2013). En referencia a la integración de la RSC en las empresas la literatura revela que muy pocas empresas la tienen integrada de manera efectiva (Bernardo, Casadesus, Karapetrovic, \& Heras, 2012; Karapetrovic \& Casadesús, 2009), a pesar de los beneficios que aporta dicha integración (Bernardo, Simon, Tarí, \& MolinaAzorín, 2015; Simon et al. 2012).

Por ello, el presente estudio parte de la existencia de diferentes motivaciones, pero queriendo detectar cuales son éstas para las empresas tecnológicas y si existen "agentes facilitadores" de su integración.

\section{Modelo conceptual e hipótesis}

En base a los apartados anteriores y analizando los trabajos previos en ellos citados referentes a la existencia de diferentes motivaciones para adoptar una estrategia de RSC en las empresas y su influencia en ella, y teniendo presente la Teoría de los Stakeholders, según la cual las empresas deben asumir responsabilidades respecto a los empleados y clientes como grupos más relevantes entre otros (Herrera et al., 2013; Hammann et al., 2009), el presente apartado trata de centrarse en cuáles son estas motivaciones en las empresas tecnológicas españolas y como una vez detectadas se puede facilitar una estrategia proactiva en materia de RSC, proponiendo las siguientes hipótesis de investigación:

H1: Existen diferentes motivaciones (tanto internas como externas) (a) que influyen positivamente en la adopción de medidas de RSC para su implantación definitiva en la empresa y (b) que influyen en la adopción de otros sistemas de gestión.

H2: (a) la existencia de sistemas de gestión normalizados -de Calidad (Q), Medioambiente (MA) y/o Seguridad y Salud Laboral (SST)- previos a la adopción de medias de RSC facilitan la implantación de la RSC y (b) ayudan (median) a su integración real en la organización.

H3: La integración de RSC de la empresa se verá afectada de manera directa y positivamente por la facilidad de implantación de las medidas de RSC.

Estas hipótesis están resumidas en el modelo conceptual reflejado en la Gráfico 1. 


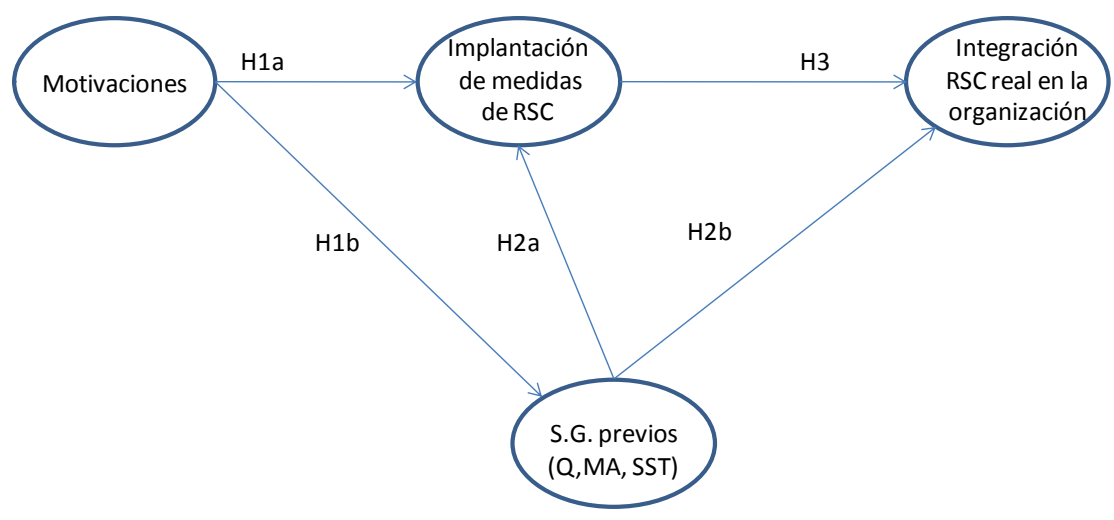

\section{Metodología}

Existe una gran variedad de métodos para la agregación de datos existentes en las ciencias sociales (Rodríguez Gutiérrez, Fuentes García, \& Sánchez Cañizares, 2013), sin embargo éstos no se aplican de manera general en el campo de la investigación de la RSC. Uno de los métodos más ampliamente utilizado es el análisis factorial, principalmente en trabajos cuya base de estudio se basa en encuestas (Rodríguez Gutiérrez et al., 2013). Para la realización del análisis se ha recurrido, en este trabajo, a un modelo de ecuaciones estructurales. Los modelos de ecuaciones estructurales son procedimientos estadísticos que permiten comprobar la medida de las hipótesis funcionales, predictivas y causales, siendo estas herramientas estadísticas multivariantes esenciales para entender muchos elementos de investigación y llevar a cabo investigación básica o aplicada en las ciencias del comportamiento, de gestión, de salud y sociales (Bagozzi \& Yi, 2011).

En los modelos de ecuaciones estructurales, se asumen relaciones más complejas (con efectos directos e indirectos) al tiempo que se trabaja con variables latentes (no observables directamente y que deben medirse a través de indicadores), llamados constructos
(Gallardo-Vázquez \& Sánchez-Hernández, 2013), lo que diferencia a estos modelos con los modelos clásicos de regresión multivariante.

Además, los modelos de ecuaciones estructurales incluyen dos niveles de análisis, el modelo de medida y el modelo estructural, (Hair , Sarstedt, Hopkins, \& Kuppelwieser, 2014). Es decir, el modelo de medida verifica cómo las constructos hipotéticos se miden en términos de las variables observadas (indicadores) y el modelo estructural examina las relaciones entre los constructos (Chen \& Chang, 2011). El modelo estructural trata de realizar un análisis similar al de la regresión pero con poder explicativo (Gallardo-Vázquez \& Sánchez-Hernández, 2013), estudiando los efectos directos e indirectos del conjunto de los constructos.

Para la formación de los constructos se ha recurrido a los siguientes indicadores (Tabla 1), basados no solo en la literatura consultada (Battaglia, Testa, Bianchi, Iraldo, \& Frey, 2014; Gallardo-Vázquez \& Sánchez-Hernández, 2014; Turyakira, Venter, \& Smith, 2014; Asif et al., 2013; Lee, Park, \& Lee, 2013) sino también en la Teoría de los Stakeholders:

Tabla 1 - Indicadores

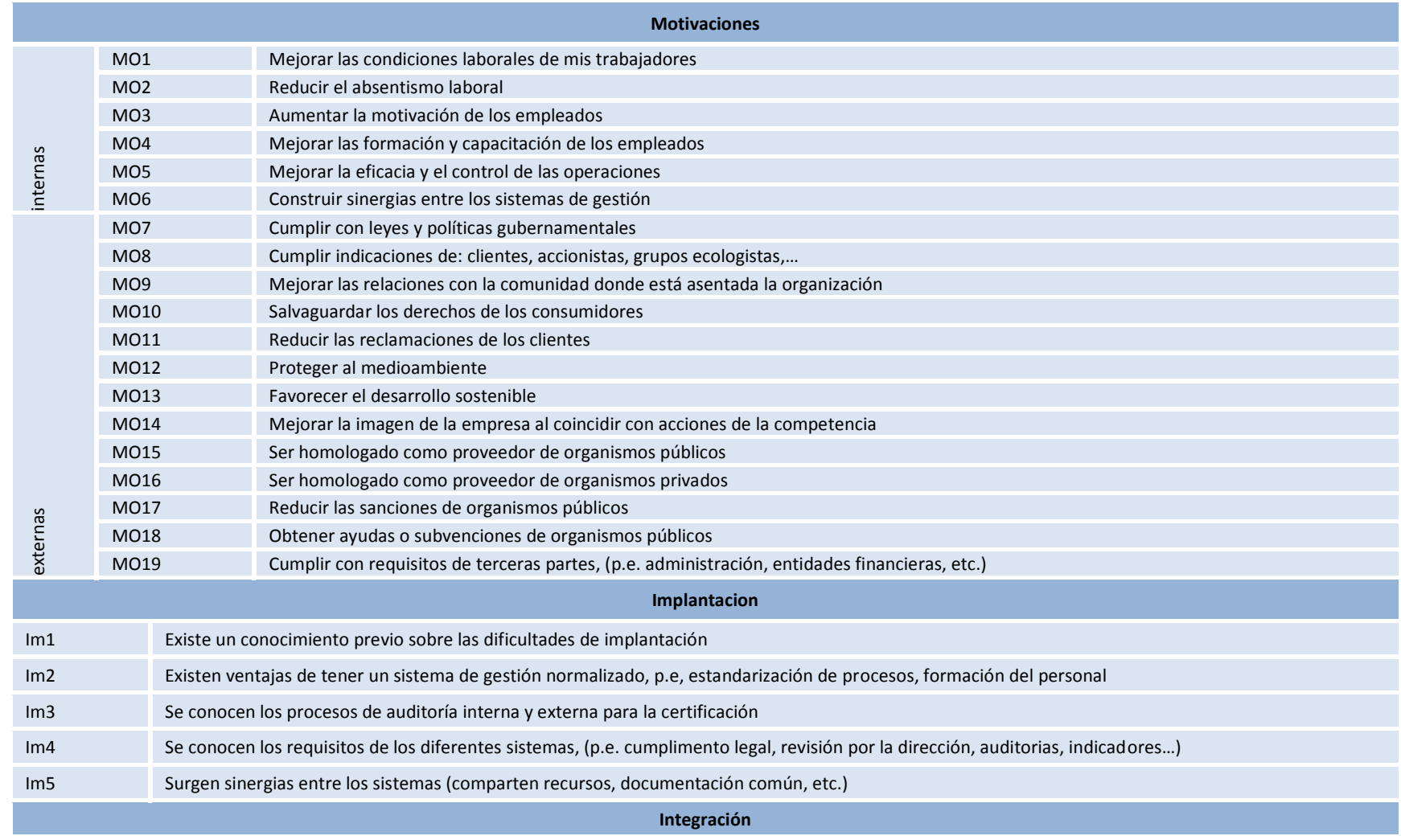




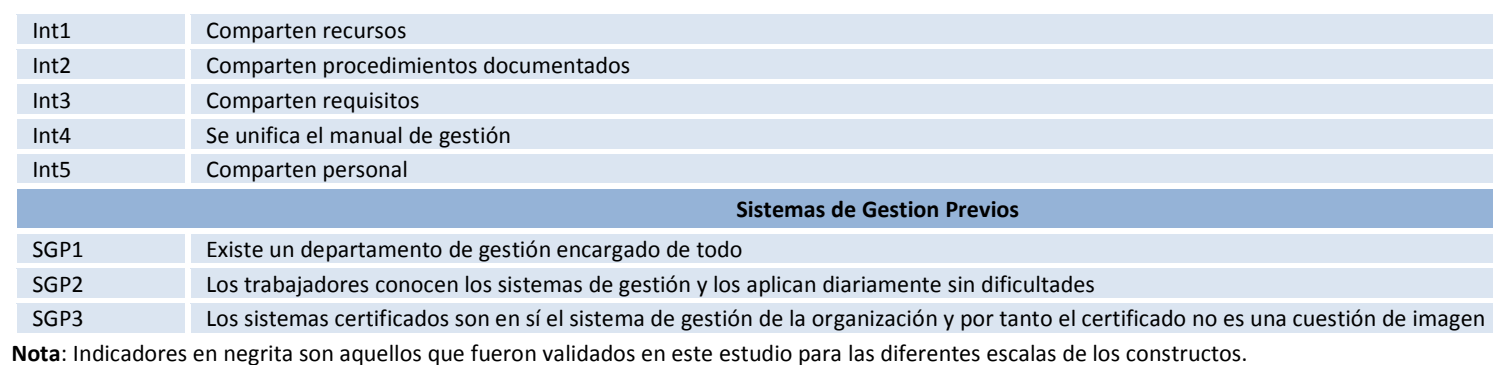

Para la elaboración de este estudio en el que se incluye tanto el examen de las relaciones entre los constructos como el análisis de las hipótesis mencionadas en la Gráfico 1, se diseñó un cuestionario especifico, utilizando la escala Likert 1-5 (1 «totalmente en desacuerdo» y 5 «totalmente de acuerdo»), debido a que un gran número de preguntas hacen referencia a cuestiones que no pueden ser cuantificadas con un valor concreto (ejemplo: Implantar medidas de RSC para aumentar la motivación del empleado). En general, el cuestionario incluía preguntas relacionadas con las motivaciones de implementación de la RSC, la integración con otros sistemas de gestión, su facilidad de integración, la adopción de una estrategia de RSC y los grupos de interés para la organización, en línea con otros estudios (GallardoVázquez \& Sánchez-Hernández, 2013; Prajogo et al., 2012; de Godos Díez, Fernández Gago, \& Cabeza García, 2012; Law \& Gunasekaran, 2012; Díez \& Gago, 2011).

Para la recogida de datos, se enviaron invitaciones mediante email para el acceso al link del cuestionario. Esta metodología tiene sus ventajas, pero no está exenta de inconvenientes, por ejemplo la tasa de respuesta se ha estimado alrededor de un $11 \%$ más baja en comparación con otros métodos más tradicionales de encuesta (Sánchez-Fernández, Muñoz-Leiva, \& Montoro-Ríos, 2012), sin embargo, autores como Hung y Law (Hung \& Law, 2011) sugieren que, a pesar de sus desventajas, los beneficios de las encuestas online superan los inconvenientes.

Para el desarrollo del trabajo empírico se ha realizado una encuesta online, recibiéndose durante el año 2014 un total de 98 cuestionarios, de los cuales se consideran validos para este estudio sobre motivaciones de la RSC, un total de 56 ya que éste es el número de empresas que habían emprendido medidas de RSC (o tenían intención de hacerlo) y por tanto tenían motivaciones sobre la RSC. La población objeto del estudio fueron 489 empresas contactadas, por tanto se tiene una tasa de respuesta válida de un $11.45 \%$ con un error del $12.35 \%$ para $\mathrm{p}=\mathrm{q}=0.5$, para una nivel de confianza del $95 \% ; z=1.96$.

La técnica elegida dentro de ecuaciones estructurales es la conocida como Partial Least Squares (PLS),por diferentes razones pues: (1) se ha utilizado previamente en las investigaciones relacionadas con la tecnología (Wang et al., 2015); (2) el uso de PLS se ha recomendado cuando el conocimiento teórico sobre un tema es escaso (Hair et al., 2014) -como es el caso de RSC y empresas tecnologías- $y$ además PLS es más adecuado para aplicaciones causales, predictivas y de construcción de teorías (análisis exploratorio) (Henseler et al., 2014; Roldán \& Sánchez-Franco, 2012); (3) PLS puede estimar modelos con indicadores reflexivos y formativos sin ningún problema de identificación (Vinzi, Chin, Henseler, \& Wang, 2010) porque trabaja con compuestos ponderados en lugar de factores (Gefen, Rigdon, \& Straub, 2011);
(4) PLS puede estimar modelos con muestras pequeñas, de hecho, los algoritmos de modelado de PLS tienden a obtener resultados con altos niveles de potencia estadística (Reinartz, Haenlein, \& Henseler, 2009), incluso cuando el tamaño de la muestra es muy modesto (Rigdon, 2014). Por lo tanto, y siguiendo a Henseler et al. (2014) utilizamos PLS como un instrumento estadístico destacable para la gestión y la investigación de las organizaciones, ya que varios estudios han indicado que el tamaño de muestra necesario está entre 30 y 100 casos si tenemos como mínimo 3 o 4 indicadores por constructo (Hair et al., 2014; Roldán \& SánchezFranco, 2012). Además, una simulación Monte Carlo realizada por Chin y Newsted (1999) muestra que PLS pueden recoger información significativa del tamaño de las muestras tan bajas como 20 (Reinartz et al., 2009). Por tanto se considera que nuestra muestra es suficiente como para llevar a cabo un análisis estadístico de modelización de ecuaciones estructurales basadas en mínimos cuadrados parciales (PLS-SEM).

\section{Análisis estadístico de los datos y resultados}

Los datos cuantitativos se recogieron a través de las respuestas que los directivos y personal altamente cualificado de empresas tecnológicas españolas- ubicadas en parques científicos y tecnológicos- realizaron a similitud de estudios previos (GallardoVázquez \& Sánchez-Hernández, 2013) (Law \& Gunasekaran, 2012).

Entre las distintas preguntas incluidas en el cuestionario se encontraban las relacionadas con las motivaciones que llevaban a los encuestados a adoptar medidas de RSC y su facilidad de implantación e integración en la empresa.

El software utilizado fue SmartPLS 2.0 M3, desarrollado por Ringle, Wende y Will en 2005. Dado que SmartPLS es un modelo de estimación y análisis PLS-SEM, utiliza el proceso de estimación en dos pasos (Chin et al. 2003), evaluando el modelo de medida y el modelo estructural (Hair et al., 2014). En primer lugar, se estima el modelo de medida, donde se determina la relación entre los indicadores y el constructo (Roldán \& Sánchez-Franco, 2012) y en segundo lugar, se realiza la estimación del modelo estructural, donde se evalúan las relaciones entre los diferentes constructos, a través de los coeficientes path, su nivel de significación $\left(R^{2}\right.$ Coefficent of determination) y la redundancia validada cruzada $\left(Q^{2}\right.$ Cross-validated redundancy) (Hair et al., 2014).

\subsection{Análisis modelo de medida}

En el modelo de medida se definen los constructos latentes que van a ser utilizados en el modelo asignándole indicadores a cada uno, por tanto, se contemplan las relaciones entre cada constructo y sus indicadores basándose en el cálculo de los componentes principales. En los modelos de medición reflexivos, este análisis se lleva a cabo con referencia a los atributos de fiabilidad individual del indicador, la fiabilidad del constructo, la validez convergente (Fornell 
\& Larcker, 1981; Tenenhaus, Vinzi, Chatelin, \& Lauro, 2005) y la validez discriminante (Hair, Sarstedt, Ringle, \& Mena, 2012).

La fiabilidad de cada elemento individual se evalúa mediante el análisis del factor de cargas estandarizadas $(\lambda)$, o correlaciones simples de los indicadores con su respectivo variable latente (Roldán \& Sánchez-Franco, 2012) o constructo (Hair et al., 2014). La fiabilidad del elemento individual es considerado adecuado cuando un indicador tiene un $\lambda$ mayor que 0.707 en su respectivo constructo (Carmines \& Zeller, 1979). Teniendo en cuenta los valores iníciales obtenidos se han eliminado del modelo, mediante procesos iterativos de depuración, los indicadores reflectivos que no cumplían el criterio de fiabilidad individual, hasta conformar el número de indicadores definitivos para cada uno de los constructos (Gallardo-Vázquez \& Sánchez-Hernández, 2014a). En este estudio, todos los indicadores reflexivos tienen cargas por encima de 0.71 (cifras en negrita en la Tabla 2).

Tabla 2 - Cargas y cargas cruzadas para el modelo de medición

\begin{tabular}{|c|c|c|c|c|}
\hline Indicador & Motivaciones & Implantación & Integración & SG previos \\
\hline MO11 & 0,712 & 0,225 & 0,324 & 0,293 \\
\hline M014 & 0,716 & 0,382 & 0,347 & 0,337 \\
\hline M015 & 0,832 & 0,279 & 0,325 & 0,252 \\
\hline MO16 & 0,843 & 0,352 & 0,346 & 0,340 \\
\hline M017 & 0,854 & 0,251 & 0,284 & 0,253 \\
\hline M018 & 0,718 & 0,200 & 0,301 & 0,186 \\
\hline M019 & 0,878 & 0,349 & 0,367 & 0,258 \\
\hline MO7 & 0,758 & 0,402 & 0,216 & 0,230 \\
\hline $\operatorname{Im} 1$ & 0,330 & 0,811 & 0,564 & 0,541 \\
\hline $\operatorname{lm} 2$ & 0,243 & 0,710 & 0,410 & 0,396 \\
\hline $\operatorname{Im} 3$ & 0,290 & 0,898 & 0,601 & 0,564 \\
\hline Im4 & 0,427 & 0,930 & 0,706 & 0,602 \\
\hline $\operatorname{Im} 5$ & 0,389 & 0,897 & 0,711 & 0,498 \\
\hline Int1 & 0,353 & 0,632 & 0,900 & 0,654 \\
\hline Int2 & 0,421 & 0,721 & 0,933 & 0,681 \\
\hline Int3 & 0,368 & 0,698 & 0,904 & 0,663 \\
\hline Int4 & 0,411 & 0,642 & 0,925 & 0,715 \\
\hline Int5 & 0,185 & 0,448 & 0,760 & 0,642 \\
\hline SGP1 & 0,330 & 0,480 & 0,741 & 0,893 \\
\hline SGP2 & 0,367 & 0,534 & 0,636 & 0,882 \\
\hline SGP3 & 0,242 & 0,640 & 0,655 & 0,915 \\
\hline
\end{tabular}

En los modelos de medida con indicadores reflexivos, se debe obtener la fiabilidad compuesta (당) (Hair et al., 2014) y el[?] de Cronbach (Castro \& Roldán, 2013), para evaluar la consistencia interna de medidas de los constructos. Se interpretan ambos valores con las directrices que ofrecieron Nunnlly y Bernstein (1994) los cuales sugieren un valor de 0,7 como punto de referencia para una fiabilidad modesta aplicable en las primeras etapas de investigación. En la presente investigación, los cuatro constructos analizados tienen una alta consistencia interna pues se superan los niveles recomendados, ya que para la fiabilidad compuesta, incluso se supera el umbral más restrictivo propuesto por Nunally y Bernstein (1994) de 0.8 (ver Tabla 3).

Para valorar la validez convergente se calcula la varianza media extraída (AVE), la cual deber ser al menos igual a 0.5 (Fornell \& Larcker, 1981) y es equivalente a la comunalidad de un constructo (Hair et al., 2014), por lo que un AVE de 0.5 demuestra que el constructo explica más de la mitad de la varianza de sus indicadores.
Por su parte, la validez discriminante representa el grado en que el constructo es empíricamente distinto de otros constructos o, en otras palabras, el constructo mide lo que realmente pretende. Dicha validez discriminante se analiza mediante dos métodos (Gefen \& Straub, 2005). Por un lado, un método para evaluar la existencia de validez discriminante es el criterio de Fornell y Larcker (1981). Este método establece que el constructo deber estar formado con más varianza de sus indicadores que cualquier otro constructo. Para corroborar este requisito, la raíz cuadrada de la AVE de cada constructo debe ser mayor que sus correlaciones con cualquier otro constructo. Esta condición se cumple para todos los constructos en relación con sus otras variables (ver Tabla 3). La segunda opción para la verificación de la validez discriminante, se realiza examinando las cargas transversales de los indicadores. Este método es considerado a menudo menos restrictivo (Henseler, Ringle, \& Sinkovics, 2009) y requiere que las cargas de cada indicador en su constructo sean más altas que las cargas cruzadas (cross-loadings) en otros constructos.

Tabla 3 - Fiabilidad compuesta (탕), coeficientes de validez convergente y discriminante

\begin{tabular}{|c|c|c|c|c|c|c|c|}
\hline & ]$_{c}$ & 国国 Cronbach & AVE & Implantación & Integración & Motivaciones & SG previos \\
\hline Implantación & 0,929 & 0,9047 & 0,727 & 0,8531 & & & \\
\hline Integración & 0,948 & 0,9305 & 0,785 & 0,7145 & 0,886 & & \\
\hline Motivaciones & 0,930 & 0,9142 & 0,626 & 0,4008 & 0,398 & 0,791 & 0,896 \\
\hline SG previos & 0,924 & 0,8776 & 0,803 & 0,6152 & 0,756 & 0,348 & \\
\hline
\end{tabular}

Nota: Elementos de la diagonal (negrita) son la raíz cuadrada de la varianza compartida entre los constructos y sus medidas (varianza media extraída). Fuera de la diagonal los elementos son las correlaciones entre constructos. Para la validez discriminante, los elementos diagonales deben ser mayores que los elementos fuera de la diagonal. 


\subsection{Análisis del modelo estructural}

Una vez que la fiabilidad y la validez del modelo de medida han sido establecidas, deben tomarse varios pasos con objeto de evaluar las relaciones hipotéticas dentro del modelo estructural (Hair et al., 2014), el cual evalúa el peso y la magnitud de las relaciones entre los distintos constructos. La bondad del ajuste del modelo es comprobada a través del análisis del estadístico de la $t$ de Student, el nivel de significación de los parámetros path (ㅁ] [?] ?]y el valor $\mathrm{R}^{2}$ para cada constructo dependiente y la prueba de StoneGeisser $\left(Q^{2}\right)$, que consiste en una validación cruzada del modelo evaluando en qué medida los parámetros estimados son útiles para predecir las variables observadas correspondientes a estos constructos (Roldán \& Sánchez-Franco, 2012).
Así en primer lugar, se aceptarán aquellos coeficientes path, y por extensión las hipótesis planteadas, que sean significativos según una distribución $t$ de Student de una cola con $n-1$ grados de libertad (Roldán \& Sánchez-Franco, 2012). Estos valores (Chin, 1998) deben ser al menos de 0,2 e idealmente superar el valor 0,3.

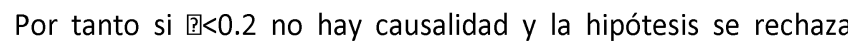
(Ramírez-Correa, 2014), en el presente caso todos los묘 son mayores de 0,2 (ver Tabla 4). De acuerdo con Hair et al. (2011) se utilizó un bootstrapping (5000 remuestras) para generar los estadísticos $t$-Student y su errores estándar, lo que nos permitió evaluar la significación estadística de los coeficientes path (Castro \& Roldán, 2013), como se observa en la Tabla 4.

Tabla 4 - Contraste de hipótesis planteadas

\begin{tabular}{|c|l|c|c|c|}
\hline H & \multicolumn{1}{|c|}{ Relaciones entre constructos } & S & Standard Error & T Statistics \\
\hline H1a & Motivaciones -> Implantación & $0,2123^{*}$ & 0,0959 & 2,2133 \\
\hline H1b & Motivaciones -> SG previos & $0,3482^{* *}$ & 0,1134 & 3,0714 \\
\hline H2a & SG previos -> Implantación & $0,5413^{* * *}$ & 0,1016 & 5,3297 \\
\hline H2b & SG previos -> Integración & $0,5091^{* * *}$ & 0,1157 & 4,3993 \\
\hline H3 & Implantación -> Integración & $0,4013 * * *$ & 0,1147 & 3,4996 \\
\hline
\end{tabular}

Nota: $\mathrm{t}(0.05,4999)=1,645158499, \mathrm{t}(0.01 .4999)=2,327094067, \mathrm{t}(0.001,4999)=3,091863446 ;{ }^{*} \mathrm{p}<0,05 .^{* *} \mathrm{p}<0,01$. $^{* * *} \mathrm{p}<$ 0,001 . ns. No significativo (basado en $\mathrm{t}(4999)$, test de una cola).

En segundo lugar, se analiza la varianza explicada. La bondad de un modelo se determina a través de la fortaleza de cada relación estructural y se analiza utilizando el valor de $\mathrm{R}^{2}$ para cada constructo dependiente. Según Falk y Miller, (Falk \& Miller, 1992), estos valores deben ser superiores a 0.1 para poder considerar que el modelo tiene suficiente capacidad predictiva. Así pues, considerando que el $\mathrm{R}^{2}$ es una medida de la exactitud del modelo (Hair et al., 2014), y que por tanto mide la cantidad de varianza del constructo que es explicada por el modelo (Serrano-Cinca et al. 2007) con los valores $0.75,0.50,0.25$, respectivamente, se describen los niveles sustanciales, moderados o débiles de la exactitud de la predicción (Hair et al., 2014; Henseler et al., 2009), aunque se considera que con valores de 0.2 se obtiene un buen poder explicativo de los constructos (Ajamieh, Benítez, Braojos, \& Gelhard, 2016). La estructura del modelo presentado y las relaciones que en él se establecen están en línea con otros estudios similares (p.e Gallardo-Vázquez \& Sánchez-Hernández, 2014b; Wang et al., 2015). Como se puede observar en la Gráfico 2 , todos los $R^{2}$ están entre el mínimo 0.1 y el 0.75 , por lo que tienen una capacidad predictiva en diferente grado.

Finalmente, el test de Stone-Giesser $\left(Q^{2}\right)$ se usa como criterio para medir la relevancia predictiva de los constructos dependientes (Roldán \& Sánchez-Franco, 2012; Wang et al., 2015) y, por tanto, es un medio para evaluar la relevancia predictiva del modelo estructural (Hair et al., 2014). Para modelos reflexivos se utiliza el índice de redundancia de validez cruzada $\left(Q^{2}\right)$ (Castro \& Roldán, 2013). Si $Q^{2}$ es mayor que 0 esto implica que el modelo tiene relevancia predictiva (Hair et al., 2014), en nuestro caso todos los $\mathrm{Q}^{2}$ obtenidos tienen signo positivo y son mayores que 0 , como se puede apreciar en la Gráfico 2.

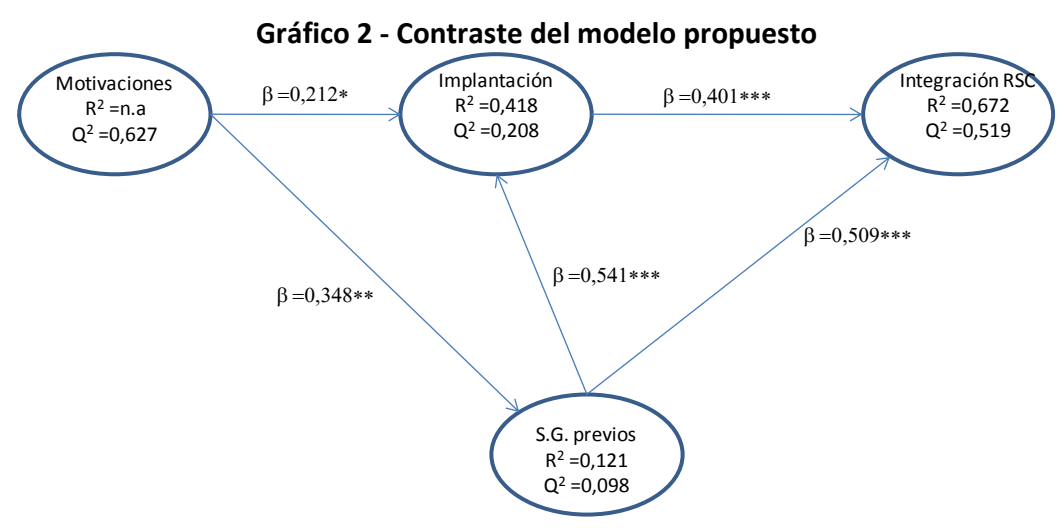

Los resultados resumidos en la Gráfico 2, confirman que el modelo estructural tiene una relevancia predictiva satisfactoria para las tres variables dependientes: los sistemas de gestión previos, la implantación de RSC y la integración real de la RSC en la organización, pudiéndose por tanto afirmar que todas las hipótesis planteadas son aceptadas.

\section{Discusión, conclusiones y limitaciones}

A la luz de los resultados se confirman las hipótesis planteadas y a pesar de que la hipótesis $\mathrm{H} 1$ a confirma que existen motivaciones para implantar la RSC en empresas tecnológicas están son sólo de 
tipo externo no existiendo de tipo interno como sugieren Graafland y Schouten (2012).

En referencia a los Sistemas de Gestión Previos $(\mathrm{H} 2)$, los resultados si confirman lo sugerido por otros autores (p.e. Battaglia et al., 2014) en cuanto a que dichos sistemas implantados en la organización son facilitadores de la integración de la RSC y tienen efectos indirectos positivos.

Una vez que se han adoptados medias de RSC, éstas son asumidas por la organización y se pueden usar para reforzar la estrategia corporativa, tal y como sugieren Isaksson et al.(2014) ya que se produce una integración real $(\mathrm{H} 3)$ y significativa, por lo que la RSC forma parte de un intangible estratégico en línea con otros estudios (Bocquet et al., 2013) que puede contribuir a la innovación y la competitividad de la empresa tecnológica.

La aplicación de medidas socialmente responsables no se traduce únicamente en un posicionamiento ético o moral por parte de las organizaciones, sino también en la generación de intangibles de elevado valor estratégico, tales como la reputación corporativa (Ramos, Manzanares, \& Gómez, 2014) o la innovación, que contribuyan al desarrollo sostenible.

En el presente estudio, el objetivo ha sido examinar las motivaciones de implantar RSC en empresas tecnológicas en mayor profundidad a través de la aplicación de un modelo predictivo y un análisis estadístico de mediación, pues, el estudio del papel de las empresas tecnológicas en la gestión ambiental y la sostenibilidad está aún en sus etapas iníciales (Wang et al., 2015).

Los resultados obtenidos muestran que las motivaciones, los sistemas de gestión previamente adoptados por las empresas y la implantación de medias de RSC están relacionados entre sí, de tal manera que los sistemas de gestión previos y la implantación de medidas de RSC median significativamente en el efecto de la integración real de la RSC en la organización.

A través del estudio realizado, se pretende cubrir el vacío detectado sobre las motivaciones en empresas tecnológicas para la implantación de medidas de RSC, ya que si bien existen estudios previos sobre motivaciones para la RSC, su integración y los resultados en empresas españolas, estos lo hacen desde un punto de vista regional (Gallardo-Vázquez \& Sánchez-Hernández, 2014a); Vintró, Fortuny, Sanmiquel, Freijo, \& Edo, 2012) o analizando un único aspecto de dicha relación (Prado-Lorenzo, Gallego-Álvarez, García-Sánchez, \& Rodríguez-Domínguez, 2008). Así pues, la ausencia de trabajos empíricos previos que analicen las motivaciones de la RSC en el sector de la tecnología en España y su integración en la empresa justificó su realización y se considera que viene a añadir un suplemento investigador a los estudios que relacionan la RSC y su integración en las empresas, pues dicha relación no se estudia con un efecto directo únicamente, sino que incorpora una relación indirecta a través de los sistemas de gestión previos como variable de mediación parcial.

La principal contribución de este trabajo ha sido demostrar la vinculación entre, las motivaciones de la RSC, las acciones de RSC y su integración en las empresas tecnológicas de manera empírica y fiable. Estos resultados son útiles para los empresarios, para motivarlos a entender porqué deben esforzarse en innovar organizativamente y en prestar atención a cuestiones relativas a la
RSC y qué pueden esperar de sus acciones. De hecho, la confirmación de las hipótesis planteadas y el buen funcionamiento del modelo nos permiten afirmar que la consideración de la RSC contribuirá en gran medida a favorecer y estimular el desarrollo sostenible de las comunidades en las que se asientan las empresas tecnológicas socialmente responsables.

Se cree además, que el presente artículo facilitará a los directivos de las empresas la identificación y el desarrollo de indicadores que permitan medir las variables involucradas en diagnosticar su posición con respecto a la RSC.

Es, por ello, importante que los empresarios y emprendedores tecnológicos conozcan sus potencialidades en RSC e integren la gestión responsable en su estrategia empresarial siendo conscientes de su relación con la competitividad de la empresa y su performance, lo que puede ser una futura línea de investigación, pues dicho análisis daría respuesta a la generación de un crecimiento inteligente y sostenible.

El presente estudio reconoce la existencia de diversas limitaciones en sus resultados y conclusiones. En primer lugar, una limitación inicial está relacionada con la noción de causalidad. Aunque la evidencia es proporcionada por la causalidad del modelo, esto en realidad no ha sido probado. El presente estudio tiene un enfoque de modelado asociativo, puesto que va dirigido más hacia la predicción de la causalidad. Mientras que la causalidad garantiza la capacidad de controlar los eventos, la asociación (predicción) sólo permite un grado limitado de control (Falk \& Miller, 1992).

En segundo lugar, otra limitación se encuentra determinada por la técnica utilizada para el modelo propuesto: ecuaciones estructurales, que asume una linealidad de las relaciones entre las variables latentes (Castro \& Roldán, 2013).

En tercer lugar, las empresas tecnológicas son organizaciones dinámicas que cambian con el tiempo. En consecuencia, la investigación futura del mismo debería medir los constructos analizados a lo largo de varios periodos de tiempo, teniendo en cuenta la dinámica para configurar las diferentes dimensiones de la RSC.

En cuarto lugar, las limitaciones del uso de características sectoriales, si bien tienen la ventaja de permitir que el sector pueda ser analizado a nivel global, muestran poca cohesión, debido al amplio espectro de empresas, desde unas dedicadas a diseñar aplicaciones para móviles hasta otras dedicadas a la seguridad y defensa. En otros subsectores en los que existe una fuerte conexión entre los miembros, podría generarse una importante línea de investigación que permitirá avanzar en nuestra comprensión del efecto de la RSC en el desempeño de la empresa tecnológica y su posible influencia en la competitividad. Finalmente, las ventajas y desventajas de la RSC dependerán de las características del contexto empírico en el que se realice el estudio.

Por último, destacar que no se ha introducido ninguna variable de control, sin embargo algunas investigaciones previas ponen de manifiesto que las grandes empresas suelen obtener mayores resultados económicos, sociales y medioambientales como consecuencia de una mayor disponibilidad de recursos, lo que les permite desarrollar un número mayor de actividades de RSC 
(Guoyou, Saixing, Chiming, Haitao, \& Hailiang, 2013) por lo que se podría utilizar el tamaño de la empresa como variable de control.

A pesar de las limitaciones indicadas anteriormente, este trabajo podría considerarse pionero, ya que representa un punto de partida para los aspectos de la RSC en cualquier empresa tecnológica y cubre el hueco detectado en la literatura. Por tanto, como futuras líneas de investigación se propone poner a prueba un modelo estructural que analizará la relación causal entre la motivación de las empresas tecnológicas hacia la RSC y la integración real en su estrategia empresarial orientado hacia la competitividad. Los resultados de ese modelo indicarán si las motivaciones de la RSC explicarían en cierto modo el éxito competitivo. Si es así, sería una estrategia interesante para las empresas tecnológicas que la desarrollen determinar (1) cuál es la intención estratégica de la RSC; (2) participar en la RSC para obtener un beneficio específico; (3) abordar la RSC como una inversión en activos intangibles; (4) centrase en una categoría específica de las partes interesadas (por ejemplo, clientes, empleados, proveedores, etc.); (5) decidir cómo comunicar la iniciativa RSC; y (6) diseñar y gestionar el proceso de toma de decisiones de la RSC.

\section{Referencias}

Ajamieh, A., Benitez, J., Braojos, J., \& Gelhard, C. (2016). IT infrastructure and competitive aggressiveness in explaining and predicting performance. Journal of Business Research, 69(10), 4667-4674. http://doi.org/10.1016/j.jbusres.2016.03.056

Asif, M., Fisscher, O. A. M., Bruijn, E. J., \& Pagell, M. (2010). Integration of management systems: A methodology for operational excellence and strategic flexibility. Operations Management Research, 3(3-4), 146-160. http://doi.org/10.1007/s12063-010-0037-z

Asif, M., Searcy, C., Zutshi, A., \& Fisscher, O. A. M. (2013). An integrated management systems approach to corporate social responsibility. Journal of Cleaner Production, 56, 7-17. http://doi.org/10.1016/j.jclepro.2011.10.034

Bagozzi, R. P., \& Yi, Y. (2011). Specification, evaluation, and interpretation of structural equation models. Journal of the Academy of Marketing Science, 4O(1), 8-34. http://doi.org/10.1007/s11747-011-0278-x

Battaglia, M., Testa, F., Bianchi, L., Iraldo, F., \& Frey, M. (2014). Corporate Social Responsibility and Competitiveness within SMEs of the Fashion Industry: Evidence from Italy and France. Sustainability, 6(2), 872-893. http://doi.org/10.3390/su6020872

Baumann-Pauly, D., Wickert, C., Spence, L. J., \& Scherer, A. G. (2013). Organizing Corporate Social Responsibility in Small and Large Firms: Size Matters. Journal of Business Ethics, 115(4), 693-705. http://doi.org/10.1007/s10551-013-1827-7

Benito-Hernández, S., \& Esteban-Sánchez, P. (2012). La influencia de las políticas de responsabilidad social y la pertenencia a redes de cooperación en el capital relacional y estructural de las microempresas. Investigaciones Europeas De Dirección y Economía De La Empresa, 18(2), 166-176.

Bernal-Conesa, J. A., De Nieves-Nieto, C., \& Briones-Peñalver, A. J. (2014). Implantación de la Responsabilidad Social en la Administración Pública: el caso de las Fuerzas Armadas Españolas. Revista De Responsabilidad Social De La Empresa, 18(III), 101-124.

Bernardo, M. (2014). Integration of management systems as an innovation: a proposal for a new model. Journal of Cleaner Production, 82, 132-142. http://doi.org/10.1016/j.jclepro.2014.06.089

Bernardo, M., Casadesus, M., Karapetrovic, S., \& Heras, I. (2012) Integration of standardized management systems: does the implementation order matter? International Journal of Operations \& Production Management, 32(3), 291-307. http://doi.org/10.1108/01443571211212583

Bernardo, M., Simon, A., Tarí, J. J., \& Molina-Azorín, J. F. (2015). Benefits of Management Systems integration: a literature review. Journal of Cleaner Production. http://doi.org/10.1016/j.jclepro.2015.01.075
Bocquet, R., Le Bas, C., Mothe, C., \& Poussing, N. (2013). Are firms with different CSR profiles equally innovative? Empirical analysis with survey data. European Management Journal, 31(6), 642-654. http://doi.org/10.1016/j.emj.2012.07.001

Boulouta, I., \& Pitelis, C. N. (2014). Who Needs CSR? The Impact of Corporate Social Responsibility on National Competitiveness. Journal of Business Ethics, 119(3), 349-364. http://doi.org/10.1007/s10551-0131633-2

Carmines, E. G., \& Zeller, R. A. (1979). Reliability and Validity Assessment. SAGE Publications.

Castro, I., \& Roldán, J. L. (2013). A mediation model between dimensions of social capital. International Business Review, 22(6), 1034-1050. http://doi.org/10.1016/j.ibusrev.2013.02.004

Chen, Y.-S., \& Chang, C.-H. (2011). Utilize structural equation modeling (SEM) to explore the influence of corporate environmental ethics: the mediation effect of green human capital. Quality \& Quantity, 47(1), 79-95. http://doi.org/10.1007/s11135-011-9504-3

Chin, W. W. (1998). Commentary: Issues and opinion on structural equation modeling. JSTOR. Retrieved from http://www.jstor.org/stable/249674

Dahlsrud, A. (2008). How corporate social responsibility is defined: an analysis of 37 definitions. Corporate Social Responsibility and Environmental Management, 15(1), 1-13. http://doi.org/10.1002/csr.132 Damanpour, F., \& Gopalakrishnan, S. (2001). The dynamics of the adoption of product and process innovations in organizations. Journal of Management Studies, 38(1), 45-65.

De Godos Díez, J. L., Fernández Gago, R., \& Cabeza García, L. (2012). Propiedad y control en la puesta en práctica de la RSC. Cuadernos De Economía y Dirección De La Empresa, 15(1), 1-11. http://doi.org/10.1016/j.cede.2011.06.002

Díez, J. L. G., \& Gago, R. F. (2011). ¿Cómo se percibe la dirección socialmente responsable por parte de los altos directivos de empresas en España? Universia Business Review, (29), 32-49.

Falk, R. F., \& Miller, N. B. (1992). A Primer for Soft Modeling (1st edition) Akron, Ohio: Univ of Akron Pr.

Fornell, C., \& Larcker, D. (1981). Evaluating Structural Equation Models with Unobservable Variables and Measurement Error. Journal of Marketing Research, 18(1), 39-50. http://doi.org/10.2307/3151312

Freeman, R.E., \& Reed, D.L. (1983). Stockholders and stakeholders: a new Perspective on Corporate Governance. California Management Review, 25(3), 88

Gallardo-Vázquez, D., \& Sánchez-Hernández, M. I. (2013). Análisis de la incidencia de la responsabilidad social empresarial en el éxito competitivo de las microempresas y el papel de la innovación. Universia Business Review, (38), 14-31.

Gallardo-Vázquez, D., \& Sánchez-Hernández, M. I. (2014a). Measuring Corporate Social Responsibility for competitive success at a regional level. Journal of Cleaner Production, 72, 14-22. http://doi.org/10.1016/j.jclepro.2014.02.051

Gallardo-Vázquez, D., \& Sánchez-Hernández, M. I. (2014b). Structural analysis of the strategic orientation to environmental protection in SMEs. $B R Q$ Business Research Quarterly, 17(2), 115-128. http://doi.org/10.1016/j.brq.2013.12.001

Gefen, D., Rigdon, E. E., \& Straub, D. (2011). An Update and Extension to SEM Guidelines for Administrative and Social Science Research. Mis Quarterly, 35(2), III-XIV.

Gefen, D., \& Straub, D. (2005). A practical guide to factorial validity using PLS-Graph: Tutorial and annotated example. Communications of the Association for Information Systems, 16(1), 5.

Graafland, J., \& Schouten, C. M.-V. der D. (2012). Motives for Corporate Social Responsibility. De Economist, 160(4), 377-396 http://doi.org/10.1007/s10645-012-9198-5

Guadamillas-Gómez, F., J Donate-Manzanares, M., \& Skerlavaj, M. (2010). The integration of corporate social responsibility into the strategy of technology-intensive firms: a case study. Zbornik Radova Ekonomskog Fakulteta u Rijeci: |vcasopis Za Ekonomsku Teoriju i Praksu, 28(1), 9-34.

Guoyou, Q., Saixing, Z., Chiming, T., Haitao, Y., \& Hailiang, Z. (2013). Stakeholders' Influences on Corporate Green Innovation Strategy: A Case Study of Manufacturing Firms in China. Corporate Social Responsibility and Environmental Management, 20(1), 1-14. http://doi.org/10.1002/csr.283 
Hair, J. F., Sarstedt, M., Ringle, C. M., \& Mena, J. A. (2012). An assessment of the use of partial least squares structural equation modeling in marketing research. Journal of the Academy of Marketing Science, 40(3), 414-433. http://doi.org/10.1007/s11747-011-0261-6

Hair Jr, J. F., Sarstedt, M., Hopkins, L., \& Kuppelwieser, V. G. (2014). Partial least squares structural equation modeling (PLS-SEM): An emerging tool in business research. European Business Review, 26(2), 106-121. http://doi.org/10.1108/EBR-10-2013-0128

Hamidi, N., Omidvari, M., \& Meftahi, M. (2012). The effect of integrated management system on safety and productivity indices: Case study; Iranian cement industries. Safety Science, 50(5), 1180-1189. http://doi.org/10.1016/j.ssci.2012.01.004

Hammann, E.-M., Habisch, A., \& Pechlaner, H. (2009). Values that create value: socially responsible business practices in SMEs - empirical evidence from German companies. Business Ethics: A European Review, 18(1), 3751. http://doi.org/10.1111/j.1467-8608.2009.01547.x

Henseler, Jorg, Dijkstra, T. K., Sarstedt, M., Ringle, C. M., Diamantopoulos, A., Straub, D. W., ... Calantone, R. J. (2014). Common Beliefs and Reality About PLS: Comments on Ronnkko and Evermann (2013). Organizational Research Methods, 17(2), 182-209. http://doi.org/10.1177/1094428114526928

Henseler, Jörg, Ringle, C. M., \& Sinkovics, R. R. (2009). The use of partial least squares path modeling in international marketing. In New Challenges to International Marketing (Vol. 20, pp. 277-319). Emerald Group Publishing Limited. Retrieved from http://www.emeraldinsight.com/doi/abs/10.1108/S14747979(2009)0000020014

Herrera, J., Larrán, M., \& Martínez-Martínez, D. (2013). Relación entre responsabilidad social y performance en las pequeñas y medianas empresas: Revisión bibliográfica. Cuadernos De Gestión, 13(2), 39-65. http://doi.org/10.5295/cdg.120360jh

Hillenbrand, C., Money, K., \& Ghobadian, A. (2013). Unpacking the Mechanism by which Corporate Responsibility Impacts Stakeholder Relationships. British Journal of Management, 24(1), 127-146. http://doi.org/10.1111/j.1467-8551.2011.00794.x

Hung, K., \& Law, R. (2011). An overview of Internet-based surveys in hospitality and tourism journals. Tourism Management, 32(4), 717-724. http://doi.org/10.1016/j.tourman.2010.05.027

Isaksson, L., Kiessling, T., \& Harvey, M. (2014). Corporate social responsibility: Why bother? Organizational Dynamics, 43(1), 64-72. http://doi.org/10.1016/j.orgdyn.2013.10.008

Janssen, C., Sen, S., \& Bhattacharya, C. (2015). Corporate crises in the age of corporate social responsibility. Business Horizons, 58(2), 183-192. http://doi.org/10.1016/j.bushor.2014.11.002

Jiménez-Zarco, A. I., Cerdán-Chiscano, M., \& Torrent-Sellens, J. (2013). Challenges and Opportunities in the Management of Science Parks: design of a tool based on the analysis of resident companies. Review of Business Management. http://doi.org/10.7819/rbgn.v15i48.1503

Jørgensen, T. H., Remmen, A., \& Mellado, M. D. (2006). Integrated management systems - three different levels of integration. Journal of Cleaner Production, 14(8), 713-722. http://doi.org/10.1016/j.jclepro.2005.04.005

Karapetrovic, S., \& Casadesús, M. (2009). Implementing environmental with other standardized management systems: Scope, sequence, time and integration. Journal of Cleaner Production, 17(5), 533-540. http://doi.org/10.1016/j.jclepro.2008.09.006

Law, K. M. Y., \& Gunasekaran, A. (2012). Sustainability development in high-tech manufacturing firms in Hong Kong: Motivators and readiness. International Journal of Production Economics, 137(1), 116-125. http://doi.org/10.1016/j.ijpe.2012.01.022

Lee, E. M., Park, S.-Y., \& Lee, H. J. (2013). Employee perception of CSR activities: Its antecedents and consequences. Journal of Business Research 66(10), 1716-1724. http://doi.org/10.1016/j.jbusres.2012.11.008

Luna Sotorrío, L., \& Fernández Sánchez, J. L. (2010). Corporate social reporting for different audiences: the case of multinational corporations in Spain. Corporate Social Responsibility and Environmental Management, 17(5), 272-283. http://doi.org/10.1002/csr.215

Melé, D., Argandoña, A., \& Sanchez-Runde, C. (2011). Facing the Crisis: Toward a New Humanistic Synthesis for Business. Journal of Business Ethics, 99(1), 1-4. http://doi.org/10.1007/s10551-011-0743-y
Melé, D., Debeljuh, P., \& Arruda, M. C. (2006). Corporate Ethical Policies in Large Corporations in Argentina, Brazil and Spain. Journal of Business Ethics, 63(1), 21-38. http://doi.org/10.1007/s10551-005-7100-y

Mendoza, S., De Nieves, C., \& Briones, A. J. (2010). Capacidades Empresariales en Responsabilidad Social y Cooperación en los Agronegocios de la Región de Murcia.

Moseñe, J. A., Burritt, R. L., Sanagustín, M. V., Moneva, J. M., \& TingeyHolyoak, J. (2013). Environmental reporting in the Spanish wind energy sector: an institutional view. Journal of Cleaner Production, 40, 199-211. http://doi.org/10.1016/j.jclepro.2012.08.023

Muñoz, R. M., Pablo, J. D. S. de, \& Peña, I. (2015). Linking corporate social responsibility and financial performance in Spanish firms. European Journa of International Management, 9(3), 368-383. http://doi.org/10.1504/EJIM.2015.069133

Nunnally, J. C., \& Bernstein, I. H. (1994). Psychometric Theory (3rd edition). New York: McGraw-Hill.

Pérez Ruiz, A., \& Rodríguez del Bosque, I. (2012). La imagen de Responsabilidad Social Corporativa en un contexto de crisis económica: E caso del sector financiero en España. Universia Business Review, (33), 1429.

Perrini, F., Russo, A., \& Tencati, A. (2007). CSR Strategies of SMEs and Large Firms. Evidence from Italy. Journal of Business Ethics, 74(3), 285-300. http://doi.org/10.1007/s10551-006-9235-x

Prado-Lorenzo, J. M., Gallego-Álvarez, I., García-Sánchez, I.-M., \& Rodríguez-Domínguez, L. (2008). Social responsibility in Spain: Practices and motivations in firms. Management Decision, 46(8), 1247-1271. http://doi.org/10.1108/00251740810901417

Prahalad, C. K., \& Ramaswamy, V. (2004). Co-creating unique value with customers. Strategy \& Leadership, 32(3), 4-9. http://doi.org/10.1108/10878570410699249

Prajogo, D., Tang, A. K. Y., \& Lai, K. (2012). Do firms get what they want from ISO 14001 adoption?: an Australian perspective. Journal of Cleaner Production, 33, 117-126. http://doi.org/10.1016/j.jclepro.2012.04.019

Quintana García, C., Benavides Velasco, C. A., \& Guzmán Parra, V. F. (2013). Capacidades de investigación y directivas: señales informativas en la salida a bolsa de las empresas de base tecnológica. Cuadernos De Economía y Dirección De La Empresa, 16(4), 270-280. http://doi.org/10.1016/j.cede.2013.06.005

Ramírez-Correa, P. (2014). Uso de internet móvil en Chile: explorando los antecedentes de su aceptación a nivel individual. Ingeniare. Revista Chilena De Ingeniería, 22(4), 560-566.

Ramos, M. I. G., Manzanares, M. J. D., \& Gómez, F. G. (2014). El efecto de papel mediador de la reputación corporativa en la relación entre la rsc y los resultados económicos. Revista De Estudios Empresariales. Segunda Época, (1). Retrieved from http://revistaselectronicas.ujaen.es/index.php/REE/article/view/1378

Ratinho, T., \& Henriques, E. (2010). The role of science parks and business incubators in converging countries: Evidence from Portugal. Technovation, 30(4), 278-290. http://doi.org/10.1016/j.technovation.2009.09.002

Reinartz, W., Haenlein, M., \& Henseler, J. (2009). An empirical comparison of the efficacy of covariance-based and variance-based SEM. International Journal of Research in Marketing, 26(4), 332-344. http://doi.org/10.1016/j.ijresmar.2009.08.001

Rigdon, E. E. (2014). Rethinking Partial Least Squares Path Modeling: Breaking Chains and Forging Ahead. Long Range Planning, 47(3), 161-167. http://doi.org/10.1016/j.Irp.2014.02.003

Rodríguez Gutiérrez, P., Fuentes García, F. J., \& Sánchez Cañizares, S. (2013). Revelación de información sobre clientes, comunidad, empleados y medio ambiente en las entidades financieras españolas a través de las memorias de responsabilidad social corporativa (2007-2010) Investigaciones Europeas De Dirección y Economía De La Empresa, 19(3), 180-187. http://doi.org/10.1016/j.iedee.2012.12.002

Roldán, J. L., \& Sánchez-Franco, M. J. (2012). Variance-Based Structura Equation Modeling: Guidelines for Using Partial Least Squares. Research Methodologies, Innovations and Philosophies in Software Systems Engineering and Information Systems, 193.

Sánchez-Fernández, J., Muñoz-Leiva, F., \& Montoro-Ríos, F. J. (2012) Improving retention rate and response quality in Web-based surveys. Computers in Human Behavior, 28(2), 507-514. http://doi.org/10.1016/j.chb.2011.10.023 
Serrano-Cinca, C., Fuertes-Callén, Y., \& Gutiérrez-Nieto, B. (2007). Online reporting by banks: a structural modelling approach. Online Information Review, 31(3), 310-332. http://doi.org/10.1108/14684520710764096

Simon, A., Karapetrovic, S., \& Casadesus, M. (2012). Evolution of Integrated Management Systems in Spanish firms. Journal of Cleaner Production, 23(1), 8-19. http://doi.org/10.1016/j.jclepro.2011.10.025

Simon, A., Karapetrovic, S., \& Casadesús, M. (2012). Difficulties and benefits of integrated management systems. Industrial Management \& Data Systems, 112(5), 828-846. http://doi.org/10.1108/02635571211232406

Spence, L. J. (2007). CSR and Small Business in a European Policy Context: The Five "C"s of CSR and Small Business Research Agenda 2007. Business and Society Review, 112(4), 533-552. http://doi.org/10.1111/j.14678594.2007.00308.x

Tenenhaus, M., Vinzi, V. E., Chatelin, Y. M., \& Lauro, C. (2005). PLS path modeling. Computational Statistics \& Data Analysis, 48(1), 159-205. http://doi.org/10.1016/j.csda.2004.03.005

Turyakira, P., Venter, E., \& Smith, E. (2014). The impact of corporate social responsibility factors on the competitiveness of small and medium-sized enterprises. South African Journal of Economic and Management Sciences, 17(2), 157-172.

Vásquez-Urriago, Á. R., Barge-Gil, A., \& Rico, A. M. (2012). Los parques científicos y tecnológicos españoles, impulsores de la cooperación en innovación. ICE: Revista De Economía, (869), 99-114.

Vásquez-Urriago, Á. R., Barge-Gil, A., Rico, A. M., \& Paraskevopoulou, E. (2014). The impact of science and technology parks on firms' product innovation: empirical evidence from Spain. Journal of Evolutionary Economics, 1-39. http://doi.org/10.1007/s00191-013-0337-1

Vázquez-Carrasco, R., \& López-Pérez, M. E. (2013). Small \& medium-sized enterprises and Corporate Social Responsibility: a systematic review of the literature. Quality \& Quantity, 47(6), 3205-3218. http://doi.org/10.1007/s11135-012-9713-4

Vilanova, M., Lozano, J. M., \& Arenas, D. (2009). Exploring the Nature of the Relationship Between CSR and Competitiveness. Journal of Business Ethics, 87(S1), 57-69. http://doi.org/10.1007/s10551-008-9812-2

Vintró, C., Fortuny, J., Sanmiquel, L., Freijo, M., \& Edo, J. (2012). Is corporate social responsibility possible in the mining sector? Evidence from Catalan companies. Resources Policy, 37(1), 118-125. http://doi.org/10.1016/j.resourpol.2011.10.003

Vinzi, V. E., Chin, W. W., Henseler, J., \& Wang, H. (2010). Handbook of Partial Least Squares: Concepts, Methods and Applications. Springer Science \& Business Media.

Von Ahsen, A. (2014). The Integration of Quality, Environmental and Health and Safety Management by Car Manufacturers - a Long-Term Empirical Study. Business Strategy and the Environment, 23(6), 395-416. http://doi.org/10.1002/bse.1791

Wang, Y., Chen, Y., \& Benitez-Amado, J. (2015). How information technology influences environmental performance: Empirical evidence from China. International Journal of Information Management, 35(2), 160170. http://doi.org/10.1016/j.ijinfomgt.2014.11.005

Recibido: 19.04.2016

Reenviado: 06.07.2016

Aceptado: 07.07.2016 